\title{
Pearls \& Oy-sters: Relapse of anti-NMDA receptor encephalitis after prior first- and second-line immunotherapy
}

Sharon Chiang, PhD, Tanu Garg, MD, Austin Hu, MD, Hitha Amin, MD, Alfredo Davalos-Balderas, MD, Isabel Alfradique-Dunham, MD, and Corey E. Goldsmith, MD

Neurology ${ }^{\circledR}$ 2018;90:936-939. doi:10.1212/WNL.0000000000005517

\section{Pearls}

- $\quad$ Relapse of anti-NMDA receptor (NMDAR) encephalitis should be considered in all patients with history of anti-NMDAR encephalitis presenting with new acute-onset encephalopathy or psychosis.

- $\quad$ CSF antibody diagnostic testing is sensitive and specific for the initial diagnosis of antiNMDAR encephalitis.

- Although CSF antibody titers correlate more closely with disease severity than serum titers, the utility of comparison to baseline or remission titers in order to diagnose relapse is still under investigation.

\section{Oy-sters}

- Long-term clinical monitoring after anti-NMDAR antibody encephalitis is necessary for appropriate diagnosis and treatment of relapse, as the interpretation of elevated antibody titers without clinical change is of unclear significance.

- Although current confirmed cases suggest a median of 2 years between the initial episode and first relapse, increasing reports suggest that longer periods of monitoring may be needed.

A 33-year-old right-handed woman with a history of anti-NMDA receptor (NMDAR) encephalitis presented with a 1-day history of vomiting and bizarre behavior in the context of recent cannabis and synthetic cannabinoid ingestion. She had been diagnosed 7 years earlier with anti-NMDAR encephalitis complicated by refractory epilepsy and treated with a 5-day course of IV methylprednisolone (IVMP), a 5-day course of IV immunoglobulin (IVIg), and 2 doses of rituximab. Her prior admission was characterized by a prolonged stay with slow clinical improvement ultimately resulting in complete resolution and no longer requiring antiepileptic medications. Anti-NMDAR immunoglobulin G ( $\mathrm{IgG}$ ) antibody was positive (titers not recorded) and $\mathrm{CT}$ chest/abdomen/pelvis, MRI pelvis, and transvaginal ultrasound were negative for malignancy. She was last seen at our institution 1.5 years prior to the current presentation without sequelae.

On current presentation, the patient was initially hemodynamically stable with intact orientation and attention. Neurologic examination was significant only for diffuse hyperreflexia. Within an hour, she became acutely obtunded and developed orofacial dyskinesias, sensory anesthesia, tachycardia, and acral hyperhidrosis with no improvement over the next 3 days. Urine toxicology was positive for cannabinoids but basic laboratory studies (complete blood count, comprehensive metabolic panel, thyroid-stimulating hormone, $\mathrm{B}_{12}$, folic acid, HIV, urinalysis) were unremarkable.
Correspondence

Dr. Goldsmith

celam@bcm.edu

From the School of Medicine (S.C.), Department of Neurology (T.G., H.A., A.D.-B., I.A.-D., C.E.G.), and Department of Internal Medicine (A.H.), Baylor College of Medicine, Houston, TX Go to Neurology.org/N for full disclosures. Funding information and disclosures deemed relevant by the authors, if any, are provided at the end of the article. 
On further workup, MRI brain with and without contrast was normal and showed neither T2 fluid-attenuated inversion recovery (FLAIR) changes nor enhancement. EEG showed diffuse slowing with no posterior dominant rhythm or epileptiform discharges. CSF showed 40 white blood cells (normal 0-5) with 90\% lymphocytes and 10\% monocytes, glucose $61 \mathrm{mg} / \mathrm{dL}$ (normal 50-80), protein $24.2 \mathrm{mg} / \mathrm{dL}$ (normal 15-45), and 0 red blood cells. CSF flow cytometry was negative for aberrant T-/B-cell populations, and cytology was negative for malignant cells. Routine CSF studies were negative for gram stain and culture, herpes simplex virus PCR, and arbovirus antibody. Acid-fast bacilli and fungal cultures were not sent due to low index of suspicion. Serum cryptococcal antigen and syphilis were negative. Repeat CT abdomen/pelvis and transvaginal ultrasound showed no teratoma or masses. CSF anti-NMDAR IgG antibody showed positive titers 1: 40 (normal 1:1).

Given the patient's lack of spontaneous clinical improvement, suggesting another etiology besides acute drug intoxication, she was started on IVMP $1 \mathrm{~g}$ daily on hospital day 3 and completed a 5-day course with no clinical improvement. She developed generalized tonic-clonic seizures on hospital day 4, requiring 1 antiepileptic medication (valproic acid). Due to minimal response to IVMP, she was treated with 5 days of IVIg $20 \mathrm{~g}$ (Privigen; CSL Behring, Berne, Switzerland) followed by IV rituximab $1 \mathrm{~g}$ (Rituxan; Biogen, Cambridge, MA) on hospital day 12 with slow clinical improvement in level of consciousness and progression to intermittent simple command following. Repeat EEG showed the presence of a posterior dominant rhythm at normal frequencies with generalized slowing anteriorly. No clear evidence of extreme delta brush was identified (figure 1). She was given a second administration of IV rituximab $1 \mathrm{~g}$ on hospital day 27, which was followed by resolution of sensory anesthesia and improvement in simple command following. She was discharged on hospital day 36 to a long-term care facility. Three-month outpatient follow-up reported complete resolution of confusion and bizarre behavior and no seizures since discharge on valproic acid. Follow-up examination was nonfocal, with intact orientation, recent/remote memory, attention, concentration, fluent speech, and comprehension to complex commands. Sensation was intact in bilateral upper and lower extremities. Repeat EEG was normal. Due to clinical improvement, no additional rituximab was given. A second repeat follow-up 6 months after admission was planned.

\section{Discussion}

We describe a case of anti-NMDAR encephalitis relapse 7 years after the initial episode in a patient with prior first- and second-line immunotherapy. Recently discovered in 2007, ${ }^{1}$ anti-NMDAR encephalitis now represents the leading cause of encephalitis in patients younger than 30 years. $^{2}$ Recognition of anti-NMDAR encephalitis is an important aspect of clinical practice, as it is responsive to immunotherapy but fatal in $7 \%{ }^{3}$ However, diagnosing anti-NMDAR encephalitis relapse is difficult, as monitoring guidelines and natural history are not yet well-characterized. We discuss current evidence on the length of long-term monitoring after the initial NMDAR encephalitis episode, examine the utility of diagnostic modalities, and point to important future directions for understanding anti-NMDAR relapse.

Figure 1 Scalp EEG shows no definite evidence of extreme delta brush

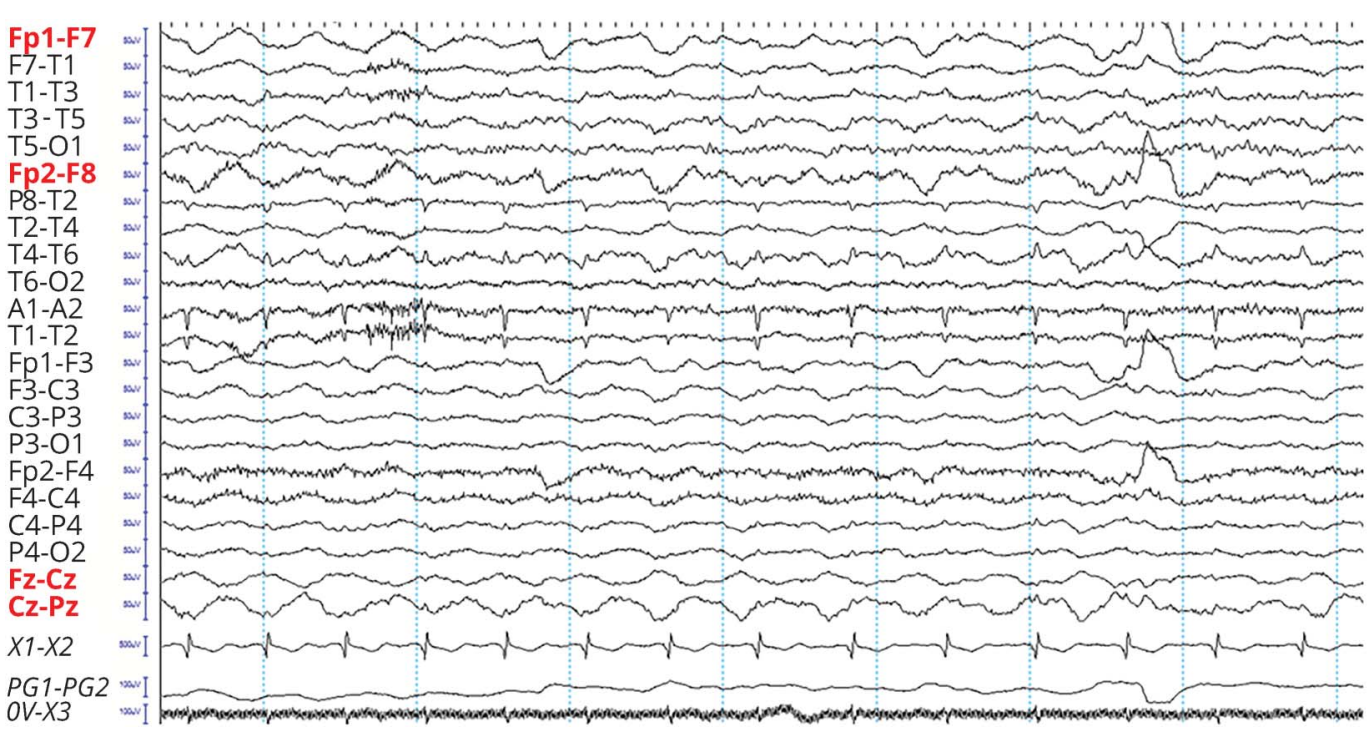

Waveforms resembling extreme delta brush were observed on repeat scalp EEG and are shown, but were neither persistent nor well-developed. Red leads indicate where these waveforms were observed. 
Anti-NMDAR encephalitis is an autoimmune encephalitis caused by IgG1/IgG3 antibodies against the NR1/NR2 subunits of the NMDAR, which most commonly affects children and young adults with or without underlying neoplasm. ${ }^{4}$ Of patients with underlying neoplasm, ovarian teratomas are most common. ${ }^{5}$ The initial presentation of antiNMDAR encephalitis is characterized by a nonspecific flu-like prodrome, followed by acute onset of neuropsychiatric symptoms. Encephalitis often progresses from psychosis, memory problems, and seizures into a state of unresponsiveness with alternating catatonia and agitation, and is often associated with orofacial/lingual dyskinesias, dissociative anesthesia, and autonomic instability. ${ }^{6}$ Brain MRI may show a nonspecific T2 or FLAIR hyperintensity of the meninges, mesial temporal lobes, or cerebral cortex, but is neither sensitive nor specific. ${ }^{4}$ EEG is of greater sensitivity and abnormal in $92 \%$ of patients, commonly showing a nonspecific generalized/frontotemporal slowing in the delta-theta range without epileptic discharges. ${ }^{4}$ Extreme delta brush, a pattern of rhythmic delta with superimposed beta activity, provides a highly specific electrographic signature. However, sensitivity is low, as it is seen in only $30 \%$ of patients after 1 and 3 days of continuous EEG. ${ }^{7}$ CSF is sensitive but not specific, with abnormalities in $95 \%$ and commonly involving lymphocytic pleocytosis with normal or mildly increased protein. ${ }^{4}$ Oligoclonal bands are present in $26 \%{ }^{4}$ Although MRI, EEG, and CSF provide useful ancillary testing, diagnosis relies on serum and CSF autoantibody testing. Treatment is often initiated empirically while antibody testing is pending (figure e-1, links. lww.com/WNL/A438).

An important question in managing patients with prior antiNMDAR encephalitis concerns the duration of long-term monitoring after the initial episode to facilitate early relapse recognition. Relapse is estimated to affect $15 \%-25 \%$ of cases, ${ }^{4,5,8}$ with first- and second-line immunotherapy significantly reducing relapse risk. ${ }^{5}$ One retrospective study of 6 patients with relapse found that all cases occurred in patients with either no immunotherapy or only first-line immunotherapy. ${ }^{8}$ In another study of 250 patients with anti-NMDAR encephalitis, 15 relapses were reported, of which only 2 received rituximab or chemotherapy. ${ }^{3}$

Current recommendations suggest long-term immunosuppression for 1 year post-treatment with mycophenolate mofetil or azathioprine to prevent relapse. ${ }^{6}$ Two studies of $6^{8}$ and $15^{3}$ relapses found a median delay of 2 years between first episode and first relapse, ${ }^{8}$ and a median delay of $1.5-5.8$ years between first episode and last relapse. ${ }^{3,8}$ Few confirmed cases have been reported of a delay $>5$ years. ${ }^{3,8}$ Our case of a relapse after 7 years, however, points to the need for longer periods of monitoring.

A second clinical question in diagnosing anti-NMDAR relapse concerns the modality of diagnosis. Serum antibody titers do not correlate reliably with disease severity, remission, or relapse. ${ }^{3}$ While both serum and CSF anti-NMDAR antibody are highly specific $(96.3 \%-100 \%)^{4}$ for the initial episode, serum antibody has a sensitivity of only $80.7 \%-89.4 \%$, whereas CSF antibody has a sensitivity of $98.5 \%-100 \%$. ${ }^{4,5,9}$

While not yet routinely used for long-term monitoring, longitudinal CSF titers may have utility for diagnosing relapse. However, comparison to baseline titers from the initial episode may not be useful. One study found an equivocal association with clinical relapse when CSF titers were compared to initial episode titers; 2 of 8 patients with relapse demonstrated titer increases, 3 demonstrated decreases, and 3 demonstrated no change. ${ }^{3}$ Comparison to remission titers may be more useful. The same study found that 4 of 6 patients had increases in CSF titers between remission and relapse, while only one patient had a decrease and one had no change. ${ }^{3}$

As these proportions are based on small samples, additional data are needed to further assess utility. However, there are practical limitations for obtaining CSF antibody titers during remission, including cost and invasiveness. Another question is whether asymptomatic patients with positive CSF antibody should be treated with immunotherapy. Our patient did not have CSF anti-NMDAR antibodies measured after her initial episode upon return to neurologic baseline. Her relapse showed a relatively low titer of 1:40 in the setting of clear and prolonged recurrence of typical symptoms. Due to practical limitations on obtaining longitudinal CSF antibody titers during remission and unclear significance in the absence of clinical change, clinical monitoring is likely sufficient, with repeat investigations initiated with recurrence of symptoms. Further data on the distribution of relapse times, as well as the natural history of antibody titers, is needed to assess the necessary minimum duration of long-term monitoring in anti-NMDAR encephalitis.

An additional consideration, given increasing reports of nonparaneoplastic NMDA encephalitis, is whether treatment should differ for paraneoplastic vs nonparaneoplastic cases. The association of ovarian teratomas with anti-NMDAR encephalitis suggests that tumor expression of antigenic NMDA receptors may drive formation of anti-NMDAR antibodies and CD8 T-cell activation, leading to loss of immune tolerance. In contrast, in nonparaneoplastic etiologies, a potentially reversible mechanism of antibody-mediated NMDAR antagonism without $\mathrm{T}$-cell involvement may be present. ${ }^{10}$ Although current guidelines do not differentiate between paraneoplastic and nonparaneoplastic causes, differences in mechanism suggest that optimal monitoring and treatment may vary between causes. Further data are needed to evaluate the relative utility of immunomodulatory therapies for different subtypes of anti-NMDAR encephalitis.

\section{Author contributions}

Dr. Chiang: acquisition of data, original manuscript draft, critical revision of the manuscript. Dr. Garg: critical revision of the manuscript. Dr. Hu: critical revision of the manuscript. Dr. Amin: critical revision of the manuscript. Dr. DavalosBalderas: critical revision of the manuscript. Dr. Alfradique- 
Dunham: critical revision of the manuscript. Dr. Goldsmith: study supervision, critical revision of the manuscript.

\section{Acknowledgment}

Jay R. Gavvala, MD, MS, provided EEG images.

\section{Study funding}

No targeted funding reported.

\section{Disclosure}

The authors report no disclosures relevant to the manuscript. Go to Neurology.org/N for full disclosures.

\section{References}

1. Dalmau J, Bataller L. Limbic encephalitis: the new cell membrane antigens and a proposal of clinical-immunological classification with therapeutic implications. Neurologia 2007;22:526-537.
2. Gable MS, Sheriff H, Dalmau J, Tilley DH, Glaser CA. The frequency of autoimmune $\mathrm{N}$-methyl-D-aspartate receptor encephalitis surpasses that of individual viral etiologies in young individuals enrolled in the California Encephalitis Project. Clin Infect Dis 2012;54:899-904.

3. Gresa-Arribas N, Titulaer MJ, Torrents A, et al. Antibody titres at diagnosis and during follow-up of anti-NMDA receptor encephalitis: a retrospective study. Lancet Neurol 2014;13:167-177.

4. Dalmau J, Gleichman AJ, Hughes EG, et al. Anti-NMDA-receptor encephalitis: case series and analysis of the effects of antibodies. Lancet Neurol 2008;7:1091-1098.

5. Titulaer MJ, Dalmau J. Seizures as first symptom of anti-NMDA receptor encephalitis are more common in men. Neurology 2014;82:550-551.

6. Dalmau J, Lancaster E, Martinez-Hernandez E, Rosenfeld MR, Balice-Gordon R. Clinical experience and laboratory investigations in patients with anti-NMDAR encephalitis. Lancet Neurol 2011;10:63-74.

7. Schmitt SE, Pargeon K, Frechette ES, Hirsch LJ, Dalmau J, Friedman D. Extreme delta brush: a unique EEG pattern in adults with anti-NMDA receptor encephalitis. Neurology 2012;79:1094-1100.

8. Gabilondo I, Saiz A, Galan L, et al. Analysis of relapses in anti-NMDAR encephalitis. Neurology 2011;77:996-999.

9. Viaccoz A, Desestret V, Ducray F, et al. Clinical specificities of adult male patients with NMDA receptor antibodies encephalitis. Neurology 2014;82:556-563.

10. Melzer N, Meuth SG, Wiendl H. Paraneoplastic and non-paraneoplastic autoimmunity to neurons in the central nervous system. J Neurol 2013;260:1215-1233.

\section{Disputes \& Debates: Rapid online correspondence}

The editors encourage comments on recent articles through Disputes \& Debates:

Access an article at Neurology.org/ $N$ and click on "COMMENT" beneath the article header. Responses will be posted within 3 business days.

Before submitting a comment to Disputes \& Debates, remember the following:

- Disputes \& Debates is restricted to comments about studies published in Neurology within the last eight weeks

- Read previously posted comments; redundant comments will not be posted

- Your submission must be 200 words or less and have a maximum of five references; reference one must be the article on which you are commenting

- You can include a maximum of five authors (including yourself)

\section{Visit the Neurology ${ }^{\circledR}$ Website at Neurology.org/N}

- More article-based content on home pages

- Streamlined menus and navigation

- Enhanced blog sections for specialty areas

- Same experience on desktop, tablet, and mobile devices

- Audio summaries of current issues

- Improved article reading experience; links more evident (pdf, analytics, social media)

- Neurology ${ }^{\circledR}$ Clinical Practice initiative "Practice Current" global surveys will be accessible across sites

f Find Neurology ${ }^{\circledR}$ on Facebook: http://tinyurl.com/neurologyfan

Follow Neurology ${ }^{\circledR}$ on Twitter: https://twitter.com/GreenJournal 


\section{Neurology}

Pearls \& Oy-sters: Relapse of anti-NMDA receptor encephalitis after prior first- and
second-line immunotherapy

Sharon Chiang, Tanu Garg, Austin Hu, et al.

Neurology 2018;90;936-939

DOI 10.1212/WNL.0000000000005517

This information is current as of May 14, 2018

\section{Updated Information \&} Services

References

Subspecialty Collections

Permissions \& Licensing

Reprints including high resolution figures, can be found at: http://n.neurology.org/content/90/20/936.full

This article cites 10 articles, 4 of which you can access for free at: http://n.neurology.org/content/90/20/936.full\#ref-list-1

This article, along with others on similar topics, appears in the following collection(s):

Autoimmune diseases

http://n.neurology.org/cgi/collection/autoimmune_diseases Encephalitis

http://n.neurology.org/cgi/collection/encephalitis

Information about reproducing this article in parts (figures,tables) or in its entirety can be found online at:

http://www.neurology.org/about/about_the_journal\#permissions

Information about ordering reprints can be found online:

http://n.neurology.org/subscribers/advertise

Neurology ${ }^{\circledR}$ is the official journal of the American Academy of Neurology. Published continuously since 1951, it is now a weekly with 48 issues per year. Copyright @ 2018 American Academy of Neurology. All rights reserved. Print ISSN: 0028-3878. Online ISSN: 1526-632X.

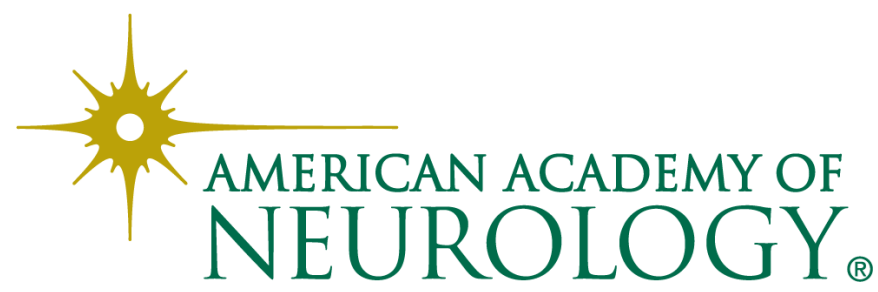

